\title{
Condition pour la fonction de convergence dans un modèle composé de particules élémentaires.
}

\author{
C. Bourrely et Roaelina Andriambololona
}

Centre de Physique Théorique, CNRS - Marseille

(Nuovo Cimento, 58 A, 205 (1968))

A la place du titre lire:

Conditions sur la fonction de convergence dans un modèle composé de particules élémentaires.

\section{Absorption Corrections for the Double Peripheral Model.}

\author{
J. I. SCHONFELDER \\ Physics Department, Imperial College - London \\ (Nuovo Cimento, $58 \mathrm{~A}, 221(1968)$ )
}

The angles $\left(\theta_{3}-\theta_{3}^{\prime}\right)$ etc.. appearing on page 228 and especially in eq. (17) should be replaced by $\cos \alpha_{3}$ etc., where $\cos \alpha_{3}$ is given by

$$
\cos \alpha_{3}=\cos \theta_{3}^{\prime} \cos \theta_{3}+\sin \theta_{3} \sin \theta_{3}^{\prime} \cos \varphi_{3}^{\prime}
$$

and similarly for $\cos \alpha_{1}$ and $\cos \alpha_{2}$.

Thus eq. (17) should read

$$
\begin{aligned}
& \left\langle\lambda_{1} \lambda_{2} \lambda_{3}\left|T^{\prime}\right| \lambda_{a} \lambda_{b}\right\rangle=\left\langle\lambda_{1} \lambda_{2} \lambda_{3}|V| \lambda_{a} \lambda_{b}\right\rangle+ \\
& +\frac{p_{a} A_{(a b)}}{16 \pi W} \int \mathrm{d} \cos \theta^{\prime}\left\langle\lambda_{1} \lambda_{2} \lambda_{3}\left|V\left(S_{a b}, S_{12}, S_{23}^{\prime}, \cos \left(\Theta_{3}-\theta^{\prime}\right), \varphi^{3}\right)\right| \lambda_{a} \lambda_{b}\right\rangle \exp \left[b_{(a b)} p_{a}^{2}\left(\cos \theta^{\prime}-1\right)\right]+ \\
& +\frac{p_{1}^{(3)} A_{(12)}}{8 W_{3}(2 \pi)^{2}} \int \mathrm{d} \cos \theta_{3}^{\prime} \mathrm{d} \varphi_{3}^{\prime} \exp \left[b_{(12)} p_{1}^{(3) 2}\left(\cos \alpha_{3}-1\right)\right]\left\langle\lambda_{1} \lambda_{2} \lambda_{3}\left|V\left(S_{c}, S_{12}^{\prime}, \cos \Theta_{3}, \cos \theta_{3}^{\prime}, \varphi_{3}^{\prime}\right)\right| \lambda_{a} \lambda_{b}\right\rangle+ \\
& \quad+\text { two sinilar terms with indices } 123 \text { cyclicly interchanged. }
\end{aligned}
$$

